\title{
Combination therapy with mTOR and PI3 kinase inhibitors is broadly synergistic in a wide variety of endometrial cancer cells
}

\author{
Shujie Yang, ${ }^{1}$ Xue Xiao, ${ }^{1}$ Xiangbing Meng, ${ }^{1,2}$ Kimberly Leslie ${ }^{1,2}$
}

Key Words: PI3K, Akt, mammalian target of rapamycin, temsirolimus, BEZ235, endometrial cancer

Background- Dysregulation of mammalian target of rapamycin (mTOR) signaling has been found in many human tumors, including endometrial cancer, and mTOR inhibitors have been utilized in clinical trials as targeted therapies. Although the mTORC1 inhibitor temsirolimus initially exhibited broad antitumor activity in preclinical models and Phase II/III clinical trials, many patients do not respond to therapy and relapse is common. Several resistance mechanisms have been hypothesized, including an increase in AKT phosphorylation after treatment with temsirolimus and subsequent activation of AKT-dependent prosurvival pathways.

Hypothesis and Approach - In order to identify a viable treatment alternative that overcomes temsirolimus-induced
AKT phosphorylation in endometrial cancer, we assessed the therapeutic benefit of dual mTOR/PI3K inhibition using a panel of endometrial cancer cell lines.

Results - Of the six drugs tested, NVPBEZ235 (dual PI3K/mTOR inhibitor) and ZSTK474 (PI3K inhibitor) successfully blocked temsirolimus-induced AKT phosphorylation. Proliferation assays revealed that in a majority of cell lines tested, BEZ235 or ZSTK474 combined with temsirolimus $(1 \mathrm{nM})$ synergistically decreased cell growth compared to cells treated with any of the agents alone. Our data suggest temsirolimus and BEZ235 inhibit different components of the AKT/mTOR signaling pathway to accomplish synergistic pathway inhibition. Specifically, temsirolimus partially blocked phosphorylation of

Department of Obstetrics \& Gynecology, ${ }^{1}$ Holden Comprehensive Cancer Center, ${ }^{2}$ The University of lowa, lowa City, lowa 52242

Corresponding author: Shujie Yang, Department of Obstetrics and Gynecology, University of lowa, 3234 MERF, 200 Hawkins Drive, lowa City, IA, 52242. Telephone(319) 335-8212 shujie-yang@uiowa.edu

This is an Open Access article distributed under the terms of the Creative Commons Attribution 3.0 Unported License (http://creativecommons.org/licenses/by/3.0), which permits unrestricted use, distribution, and reproduction in any medium, provided the original work is properly cited. 
AACR 102nd Annual Meeting, April 2 - 6, 2011,

Orlando, Florida

mTOR and completely abolished phosphorylation of $\mathrm{p} 70 \mathrm{~S} 6 \mathrm{~K}$ and its downstream substrate, rS6, at low (1 $\mathrm{nM}$ ) concentrations. In contrast, BEZ235 inhibited 4E-BP1 phosphorylation, another substrate for mTOR, whereas temsirolimus had no effect on 4E-BP1 phosphorylation. Furthermore, G0/G1 cell cycle arrest and up-regulation of p27 was observed after combination treatment of temsirolimus and BEZ235. Strikingly, cell death occurred through autophagy and not apoptosis. Finally, molecular profiling revealed that elevated basal levels of AKT phosphorylation and a loss of the tumor suppressor PTEN correlated with sensitivity to combinatorial treatment.
Conclusions - While previous studies have established that endometrial cancer cells are responsive to drugs that inhibit PI3K or mTOR, we observed a novel synergistic effect when the specific inhibitors were combined. In particular, the mechanism of synergy is through blocking two arms of the $\mathrm{PI} 3 \mathrm{~K} / \mathrm{mTOR}$ signaling pathways: p70S6K and rS6 by temsirolimus with high efficacy and AKT and 4E-BP1 by BEZ235. Combined inhibition of mTOR and PI3K is necessary for therapeutic efficacy to abrogate the increased signaling through AKT that occurs with mTOR inhibition alone. Loss of PTEN and baseline AKT phosphorylation appeared to be the most sensitive predictors for response to the $\mathrm{PI} 3 \mathrm{~K} / \mathrm{mTOR}$ inhibitor combination therapy.

Combination therapy in endometrial cancer 